Article

\title{
Triple-Frequency GPS Un-Differenced and Uncombined PPP Ambiguity Resolution Using Observable-Specific Satellite Signal Biases
}

\author{
Gen Liu ${ }^{1}$, Fei Guo ${ }^{2, *}$, Jian Wang ${ }^{1}$, Mingyi Du ${ }^{1}$ and Lizhong $Q u^{1}$ \\ 1 School of Geomatics and Urban Spatial Informatics, Beijing University of Civil Engineering and Architecture, \\ 15 Yongyuan Road, Daxing, Beijing 102616, China; liugen@bucea.edu.cn (G.L.); \\ wangjian@bucea.edu.cn (J.W.); dumingyi@bucea.edu.cn (M.D.); qulizhong@bucea.edu.cn (L.Q.) \\ 2 School of Geodesy and Geomatics, Wuhan University, 129 Luoyu Road, Wuhan 430079, China \\ * Correspondence: fguo@whu.edu.cn
}

Received: 19 May 2020; Accepted: 9 July 2020; Published: 18 July 2020

\begin{abstract}
The new generations of global navigation satellite system (GNSS) space vehicles can transmit three or more frequency signals. Multi-frequency observations bring a significant improvement to precise point positioning ambiguity resolution (PPP AR). However, the multi-frequency satellite code and phase biases need to be properly handled before conducting PPP AR. The traditional satellite bias correction methods, for example, the commonly used differential code biases (DCB), are limited to the dual-frequency ionosphere-free (IF) case and become more and more difficult to extend to multi-GNSS and multi-frequency cases. In this contribution, we propose the observable-specific signal bias (OSB) correction method for un-differenced and uncombined (UDUC) PPP AR. The OSB correction method, which includes observable-specific satellite code and phase bias correction, can directly apply kinds of OSBs to GNSS original observation data, thus, it is more appropriate for multi-GNSS and multi-frequency cases. In order to verify the performance of multi-frequency UDUC-PPP AR based on the OSB correction method, triple-frequency GPS observation data provided by 142 Multi-GNSS Experiment (MGEX) stations were used to estimate observable-specific satellite phase biases at the PPP service end and some of them were also used to conduct AR at the PPP user end. The experiment results showed: the averaged time-to-first-fix (TTFF) of triple-frequency GPS kinematic UDUC-PPP AR with observable-specific satellite code bias (SCB) corrections could reach about 22 min with about $29 \%$ improvement, compared with that without observable-specific SCB corrections; TTFF of triple-frequency static UDUC-PPP AR with observable-specific phase-specific time-variant inter-frequency clock bias (IFCB) corrections took about $15.6 \mathrm{~min}$ with about $64.3 \%$ improvement, compared with that without observable-specific IFCB corrections.
\end{abstract}

Keywords: global positioning system (GPS); precise point positioning (PPP); triple-frequency ambiguity resolution; un-differenced and uncombined; observable-specific signal bias (OSB)

\section{Introduction}

As known to all, the new generations of global navigation satellite system (GNSS) space vehicles can transmit three or more frequency signals. The global positioning system (GPS) has successively launched Block I, Block II, Block IIA, Block IIR and Block IIM satellites, and the modern generation of Block-IIF satellites can transmit the third civil signal L5 signal, in addition to the existing L1 and L2 signals [1]. The Europe Galileo system can provide five frequency navigation signals (E1, E5, E5a, E5b and E6) for commercial and civilian use. The Chinese BeiDou Navigation Satellite System (BDS) has provided B1, B2 and B3 signals. The extra frequency signals have been demonstrated to bring significant improvement to the time-to-first-fix (TTFF) and positioning accuracy of precise point 
positioning ambiguity resolution (PPP AR) [2-8]. However, it is essential that the code and phase biases on each frequency are properly handled as a prerequisite of conducting multi-frequency PPP AR. This is mainly because the code biases could reduce the accuracy of pseudo-range observations and the phase biases could not only affect carrier-phase observations but also destroy the un-difference integer ambiguity. Correct calibration of these biases could undoubtedly improve the accuracy of observation data as well as restore the un-difference integer ambiguity. In general, the code and phase biases at the satellite end need to be handled, whereas the receiver-related biases, which can be canceled by the approach of single-differences between satellites (SD), do not impact on the performance of PPP AR [9]. Therefore, the current study of the code and phase biases for PPP AR focuses on the satellite code and phase biases.

Satellite code biases (SCB) on each pseudo-range frequency signal are hard to be directly calculated or unbiasedly estimated based on the un-differenced and uncombined (UDUC) PPP model. Therefore, differential code biases (DCB), which can be obtained by differencing pseudo-range observations on two different frequency signals, are commonly used as an alternative. The types of DCB include intra-frequency code bias such as GPS P1-C1, C1C-C1W or C2W-C2X bias, as well as inter-frequency code bias such as GPS P1-P2, C1W-C2W or C1C-C5Q bias [10-12]. PPP users should correct SCB with the corresponding DCB products when using observations differing from the conventional reference signal of International GNSS Service (IGS) precise satellite clock offset products [13]. This differential SCB correction method is very suitable for the traditional dual-frequency ionosphere-free PPP model. However, in the face of a steadily growing variety of GNSS signals and observables, the differential SCB correction method is hard and not convenient to extend to multi-GNSS and multi-frequency observations. Schaer [14] and Villiger et al. [15] proposed the concept of observable-specific SCB which could directly interact with GNSS original observations. This innovation can greatly facilitate multi-GNSS and multi-frequency SCB correction and also can apply to any PPP models. However, the concrete correction strategy and the performance of PPP AR with observable-specific SCB need to be further studied.

Satellite phase biases (SPB) on each carrier-phase frequency signal include the time-invariant and time-variant parts $[16,17]$. The time-invariant parts of satellite phase biases, which are commonly defined as uncalibrated phase delays (UPD) or just SPB, are respectively absorbed into each frequency float ambiguity parameters when conducting float UDUC-PPP [18]. The classical SPB correction strategy for example is commonly to directly apply UDUC-SPB products to correct float ambiguity parameters when conducting UDUC-PPP AR at the user end [4,19-21]. However, the disadvantage of the classical SPB correction strategy is that the form of SPB products commonly depends on the form of float ambiguities. In other words, one form of SPB product can only apply to the specific float ambiguities. Therefore, in order to conveniently conduct multi-frequency and multi-GNSS PPP $\mathrm{AR}$, it is necessary to study an observable-specific SPB correction strategy, which is similar to the observable-specific SCB correction strategy.

The time-variant satellite phase biases vary cyclically from day to day with respect to Sun-satellite-Earth geometry [22]. For dual-frequency (L1/L2) UDUC-PPP, one part of time-variant satellite phase biases can be absorbed into ionospheric delay parameters, and another part of time-variant satellite phase biases can be offset by current (IGS) precise clock products, which contain a L1/L2 IF combination of time-variant satellite phase biases. In other words, time-variant satellite phase biases can be ignored for dual-frequency (L1/L2) UDUC-PPP. However, when using the additional L5 observations to conduct triple-frequency UDUC-PPP, the time-varying satellite phase biases on the L5 frequency cannot be offset by L1/L2 IGS precise clock products. Therefore, the inconsistency between the time-varying phase biases on the L5 frequency and the time-varying phase biases included in the L1/L2 IGS precise clock products, which is commonly defined as phase-specific inter-frequency clock bias (PIFCB or IFCB), need to be additionally considered. IFCB also includes an observable-specific or IF form, which are respectively applicable to a triple-frequency UDUC-PPP model and a triple-frequency IF-PPP model [23]. According to the current study, a triple-frequency 
GPS float PPP with IFCB correction can improve the positioning accuracy and reduce the convergence time [17,23-25]. However, the performance of GPS triple-frequency PPP AR with observable-specific IFCB has not been investigated so far.

In this paper, we aim at proposing an observable-specific signal bias (OSB) correction method for UDUC-PPP AR, and meanwhile, the performance of triple-frequency GPS UDUC-PPP AR with OSB is also investigated. To achieve the goals, we first provide a summary of the current satellite code and phase biases based on a triple-frequency UDUC-PPP observation model. Thereafter, the observable-specific SCB, SPB and IFCB corrections for triple-frequency UDUC-PPP AR are discussed. Subsequently, we use real GPS triple-frequency observation data to conduct an experiment to validate the performance of triple-frequency UDUC-PPP AR with OSB. Finally, the main points and conclusions are summarized.

\section{Methods}

Considering time-variant and time-invariant code and phase biases, the observation equation for original pseudo-range and carrier-phase observations on each frequency can be shown as follows [17,23]:

$$
\begin{gathered}
P_{r, n}^{s}=\rho_{r}^{s}+t_{r}-t^{s}+\gamma_{n} \cdot I_{r, 1}^{s}+m_{r}^{s} \cdot z w d_{r}+\left(d_{r, n}+\Delta d_{r, n}\right)-\left(d_{n}^{s}+\Delta d_{n}^{s}\right)+\varepsilon_{r, n}^{s} \\
L_{r, n}^{s}=\rho_{r}^{s}+t_{r}-t^{s}-\gamma_{n} \cdot I_{r, 1}^{s}+m_{r}^{s} \cdot z w d_{r}+\lambda_{n} \cdot N_{r, n}^{s}+\lambda_{n} \cdot\left(b_{r, n}+\Delta b_{r, n}\right)-\lambda_{n}\left(b_{n}^{s}+\Delta b_{n}^{s}\right)+\xi_{r, n}^{s}
\end{gathered}
$$

where $n=1,2,3$ refers to the frequency number; $\rho_{r}^{s}$ is the geometric distance between the phase centers of satellite $s$ and receiver $r$ antennas $(\mathrm{m}) ; t_{r}$ and $t^{s}$ are physical receiver and satellite clock offsets, respectively $(\mathrm{m}) ; I_{r, 1}^{s}$ is the slant ionospheric delay on the L1 frequency $(\mathrm{m}) ; \gamma_{n}$ is the frequency-dependent multiplier factor at frequency $n$, which can be expressed as $\gamma_{n}=\frac{f_{1}^{2}}{f_{n}^{2}}$ and $f$ is the frequency; $z w d_{r}$ denotes the wet troposphere delay at zenith with the mapping function $m_{r}^{s}$ $(\mathrm{m}) ; \lambda_{n}$ is the wavelength of the phase measurement on the frequency band $\mathrm{n}(\mathrm{m}) ; d_{r, n}$ and $d_{n}^{s}$ denote frequency-dependent time-invariant receiver and satellite code biases, respectively (m); $\Delta d_{r, n}$ and $\Delta d_{n}^{s}$ denote frequency-dependent time-variant receiver and satellite code biases, respectively $(\mathrm{m}) ; b_{r, n}$ and $b_{n}^{s}$ are frequency-dependent time-invariant phase biases at receiver and satellite sides (cycles); $\Delta b_{r, n}$ and $\Delta b_{n}^{s}$ are frequency-dependent time-variant phase biases at receiver and satellite sides (cycles); $N_{r, n}^{s}$ is the integer ambiguity on each frequency (cycles); $\varepsilon_{r, n}^{s}$ and $\xi_{r, n}^{s}$ are the sum of noise and multipath error for code and carrier phase observations (m). It should be noted that the satellite and receiver antenna phase center offsets (PCOs) and variations (PCVs), relativistic effects, slant hydrostatic troposphere delay, tidal loadings and phase wind-up (only for carrier phase) have been corrected with the existing models.

\subsection{Triple-Frequency UDUC-PPP AR Model}

In general, the time-variant code biases are far less than the pseudo-range measurement noise, while time-variant phase biases at the receiver sides can be absorbed by the receiver clock parameter, and its influence can be eliminated by the approach of single differences between satellites when conducting PPP ambiguity resolution, and as a result, the two kinds of biases can be safely neglected $[23,26]$. Therefore, according to Equations (1) and (2), a GPS triple-frequency UDUC-PPP observation model for pseudo-range and carrier-phase measurements can be simplified as:

$$
\begin{gathered}
\left\{\begin{array}{l}
P_{r, 1}^{s}=\rho_{r}^{s}+t_{r}-t^{s}+\gamma_{1} \cdot I_{r, 1}^{s}+m_{r}^{s} \cdot z w d_{r}+d_{r, 1}-d_{1}^{s}+\varepsilon_{r, 1}^{s} \\
P_{r, 2}^{s}=\rho_{r}^{s}+t_{r}-t^{s}+\gamma_{2} \cdot I_{r, 1}^{s}+m_{r}^{s} \cdot z w d_{r}+d_{r, 2}-d_{2}^{s}+\varepsilon_{r, 2}^{s} \\
P_{r, 5}^{s}=\rho_{r}^{s}+t_{r}-t^{s}+\gamma_{5} \cdot I_{r, 1}^{s}+m_{r}^{s} \cdot z w d_{r}+d_{r, 5}-d_{5}^{s}+\varepsilon_{r, 5}^{s}
\end{array}\right. \\
\left\{\begin{array}{l}
L_{r, 1}^{s}=\rho_{r}^{s}+t_{r}-t^{s}-\gamma_{1} \cdot I_{r, 1}^{s}+m_{r}^{s} \cdot z w d_{r}+\left(N_{r, 1}^{s}+b_{r, 1}-d_{1}^{s}\right)-\Delta d_{1}^{s}+\xi_{r, 1}^{s} \\
L_{r, 2}^{s}=\rho_{r}^{s}+t_{r}-t^{s}-\gamma_{2} \cdot I_{r, 1}^{s}+m_{r}^{s} \cdot z w d_{r}+\left(N_{r, 2}^{s}+b_{r, 2}-d_{2}^{s}\right)-\Delta d_{2}^{s}+\xi_{r, 2}^{s} \\
L_{r, 5}^{s}=\rho_{r}^{s}+t_{r}-t^{s}-\gamma_{5} \cdot I_{r, 1}^{s}+m_{r}^{s} \cdot z w d_{r}+\left(N_{r, 5}^{s}+b_{r, 5}-d_{5}^{s}\right)-\Delta d_{5}^{s}+\xi_{r, 5}^{s}
\end{array}\right.
\end{gathered}
$$


where $P_{r, 1}^{s}, P_{r, 2}^{s}$ and $P_{r, 5}^{s}$ denote the original pseudo-range measurements on GPS L1, L2 and L5 signals, while $L_{r, 1}^{s}, L_{r, 2}^{s}$ and $L_{r, 5}^{s}$ are original carrier-phase measurements, correspondingly; the other symbols on corresponding frequency signals have the same meaning as those in Equations (1) and (2). As discussed above, the GPS satellite clock offset products are computed by ionosphere-free (IF) combination of phase (L1/L2) and code (P1/P2) observations. Therefore, the GPS satellite clock products which contain the time-invariant and time-variant bias can be described as:

$$
\bar{t}^{s}=t^{s}-\left(d_{I F_{12}}^{s}+\Delta b_{I F_{12}}^{s}\right)
$$

with

$$
\left\{\begin{array}{l}
\alpha_{12}=f_{1}^{2} /\left(f_{1}^{2}-f_{2}^{2}\right) \\
\beta_{12}=-f_{2}^{2} /\left(f_{1}^{2}-f_{2}^{2}\right) \\
d_{I F_{12}}^{s}=\alpha_{12} \cdot d_{1}^{s}+\beta_{12} \cdot d_{2}^{s} \\
\Delta b_{I F_{12}}^{s}=\alpha_{12} \cdot \Delta b_{1}^{s}+\beta_{12} \cdot \Delta b_{2}^{s}
\end{array}\right.
$$

where $\bar{t}^{s}$ denote the IGS satellite clock product; $d_{I F_{12}}^{s}$ denote time-invariant IF combination satellite code biases; $\Delta b_{I F_{12}}^{s}$ denote time-variant IF satellite phase biases; $\alpha_{12}$ and $\beta_{12}$ are the IF combination factor. After using the IGS satellite clock products, the satellite clock parameter in (3) and (4) can be removed. Based on S-system theory [19], the full rank linear observation equations can be reparameterized as:

$$
\begin{aligned}
\left\{\begin{array}{l}
\bar{P}_{r, 1}^{s}=\mu \cdot X+t_{r, 12}+\gamma_{1} \cdot \bar{I}_{r, 1}^{s}+m_{r}^{s} \cdot z w d_{r}+\Delta b_{1}^{s}+\varepsilon_{r, 1}^{s} \\
\bar{P}_{r, 2}^{s}=\mu \cdot X+t_{r, 12}+\gamma_{2} \cdot \bar{I}_{r, 1}^{s}+m_{r}^{s} \cdot z w d_{r}+\Delta b_{2}^{s}+\varepsilon_{r, 2}^{s} \\
\bar{P}_{r, 5}^{s}=\mu \cdot X+t_{r, 12}+\gamma_{5} \cdot \bar{I}_{r, 1}^{s}+m_{r}^{s} \cdot z w d_{r}+I F B_{r}^{s}+\gamma_{5} \cdot \beta_{12} \cdot\left(\Delta b_{1}^{s}-\Delta b_{2}^{s}\right)+\Delta b_{I F_{12}}^{s}+\varepsilon_{r, 5}^{s}
\end{array}\right. \\
\qquad \begin{array}{l}
\bar{L}_{r, 1}^{s}=\mu \cdot X+t_{r, 12}-\gamma_{1} \cdot \bar{I}_{r, 1}^{s}+m_{r}^{s} \cdot z w d_{r}+\bar{N}_{r, 1}^{s}+\xi_{r, 1}^{s} \\
\bar{L}_{r, 2}^{s}=\mu \cdot X+t_{r, 12}-\gamma_{2} \cdot \bar{I}_{r, 1}^{s}+m_{r}^{s} \cdot z w d_{r}+\bar{N}_{r, 2}^{s}+\xi_{r, 2}^{s} \\
\bar{L}_{r, 5}^{s}=\mu \cdot X+t_{r, 12}-\gamma_{5} \cdot \bar{I}_{r, 1}^{s}+m_{r}^{s} \cdot z w d_{r}+\bar{N}_{r, 5}^{s}+I F C B^{s}+\xi_{r, 5}^{s}
\end{array}
\end{aligned}
$$

With

$$
\left\{\begin{array}{l}
D C B_{r, 12}=d_{r, 2}-d_{r, 1}, D C B^{s, 12}=d_{2}^{s}-d_{1}^{s} \\
d_{r, I F_{12}}=\alpha_{12} d_{r, 1}+\beta_{12} d_{r, 2} \\
\bar{I}_{r, 1}^{s}=I_{r, 1}^{s}-\beta_{12}\left(D C B_{r, 12}-D C B^{s, 12}+\Delta b_{1}^{s}-\Delta b_{2}^{s}\right) \\
t_{r, 12}=t_{r}+d_{r, I F_{12}} \\
\bar{N}_{r, 1}^{s}=-\gamma_{1} \cdot \beta_{12} \cdot\left(D C B_{r, 12}-D C B^{s, 12}\right)-d_{r, I F_{12}}+d_{I F_{12}}^{s}+\lambda_{1} \cdot\left(N_{r, 1}^{s}+b_{r, 1}-b_{1}^{s}\right) \\
\bar{N}_{r, 2}^{s}=-\gamma_{2} \cdot \beta_{12} \cdot\left(D C B_{r, 12}-D C B^{s, 12}\right)-d_{r, I F_{12}}+d_{I F_{12}}^{s}+\lambda_{2} \cdot\left(N_{r, 2}^{s}+b_{r, 2}-b_{2}^{s}\right) \\
\bar{N}_{r, 5}^{s}=-\gamma_{5} \cdot \beta_{12} \cdot\left(D C B_{r, 12}-D C B^{s, 12}\right)-d_{r, I F_{12}}+d_{I F_{12}}^{s}+\lambda_{5} \cdot\left(N_{r, 5}^{s}+b_{r, 5}-b_{5}^{s}\right) \\
I F B_{r}^{s}=\gamma_{5} \cdot \beta_{12} \cdot\left(D C B_{r, 12}-D C B^{s, 12}\right)-d_{r, I F_{12}}+d_{I F_{12}}^{s}+d_{r, 5}-d_{5}^{s} \\
I F C B^{s}=\Delta b_{I F_{12}}^{s}-\Delta b_{5}^{s}+\gamma_{5} \cdot \beta_{12} \cdot\left(\Delta b_{1}^{s}-\Delta b_{2}^{s}\right)
\end{array}\right.
$$

where $\bar{P}$ and $\bar{L}$ denote the OMC (observation minus computed) values of pseudo-range and carrier-phase observations; $\mu$ denotes the vector of line of sight; $X$ denotes the three-dimensional coordinates of the station; and $t_{r, 12}$ denotes IF receiver clock offset. $D C B_{r, 12}$ and $D C B^{s, 12}$ are the L1/L2 DCB at the receiver and satellite sides, respectively. $\bar{I}_{r, 1}^{s}$ is the ionospheric delay on the L1 frequency, lumped with L1/L2 DCB and time-variant phase biases; $z w d_{r}$ denotes the wet troposphere delay at zenith with the mapping function $m_{r}^{s}$; IFB $B_{r}^{s}$ denotes the time-invariant inter-frequency code bias for each satellite; $\bar{N}_{r, 1}^{s}, \bar{N}_{r, 2}^{s}$ and $\bar{N}_{r, 5}^{S}$ are reparameterized float ambiguities for each frequency; $I F C B^{s}$ refers to the phase-specific time-variant inter-frequency clock bias (IFCB). 


\subsection{Observable-Specific Satellite Code Biases}

As discussed above, satellite code biases, which are considered time-invariant biases in a period of time, are commonly corrected to pseudo-range observations using DCB products. The DCB products of P1-P2, P1-C1 and P2-C2, which release by CODE, are produced based on the RINEX 2 observations of dual-frequency GPS and GLONASS (available at ftp://ftp.aiub.unibe.ch/CODE/). With multi-GNSS and multi-frequency signals observed, DLR, IGG and CODE use multi-GNSS experiment (MGEX) stations to produce DCB products which are based on RINEX 3 observations of multi-GNSS and multi-frequency (available at: ftp://cddis.gsfc.nasa.gov/pub/gps/products/mgex/dcb/). The current code bias types for GPS signals are summarized in Table 1. However, it is noted that no standard choice and usage of DCB products have been recommended by the IGS Bias and Calibration Working Group (BCWG) [12].

Table 1. Current code bias types for global position system (GPS) signals.

\begin{tabular}{llll}
\hline Observation Format & Code Bias Type & DCB Type & Institution \\
\hline GPS RINEX 2 & Inter-frequency code bias & P1-P2 & CODE \\
& Intra-frequency code bias & P1-C1 & \\
\hline GPS RINEX 3 & P2-C2 & IGG/DLR/CODE \\
& & C1W-C2W & \\
& C1C-C1W & \\
& C1C-C2W & \\
& C1C-C5Q & \\
& Intra-frequency code bias & C2W-C2 $2 L$ & \\
& C2W-C2S & \\
& & C2W-C2X & \\
\hline
\end{tabular}

DCB products cannot be used directly to calibrate the UDUC pseudo-range measurements on each frequency because they represent relative inter-frequency or intra-frequency code bias instead of the absolute code bias on each frequency. Although only the relative relation between different satellite code biases can be obtained, we can still recover their absolute format by introducing a constraint condition. According to Equations (6) and (9), we can write the following equation system:

$$
\left\{\begin{array}{l}
D C B_{C 1 W-C 2 W}^{s}=d_{C 1 W}^{s}-d_{C 2 W}^{s} \\
d_{I F_{C 1 W C 2 W}}^{s}=\alpha_{12} \cdot d_{C 1 W}^{s}+\beta_{12} \cdot d_{C 2 W}^{s}
\end{array}\right.
$$

where $D C B_{C 1 W-C 2 W}^{s}$ denotes C1C-C1W satellite DCB; $d_{C 1 W}^{s}$ and $d_{C 2 W}^{s}$ denote satellite code biases on $\mathrm{C} 1 \mathrm{~W}$ and $\mathrm{C} 2 \mathrm{~W}$ pseudo-range observations, respectively; and $d_{I F \mathrm{C}_{1 W C 2 W}}^{\mathrm{S}}$ denotes $\mathrm{C} 1 \mathrm{~W} / \mathrm{C} 2 \mathrm{~W}$ IF combination satellite code biases. In order to be consistent with an IGS clock product relying on the same definition, the constraint condition can be expressed as [14]:

$$
d_{I F_{C 1 W C 2 W}}^{s}=0
$$

where $d_{I F_{C 1 W C 2 W}}^{s}$ is supposed as 0 value; then, the absolute $\mathrm{C} 1 \mathrm{~W}$ and $\mathrm{C} 2 \mathrm{~W}$ satellite code bias can be derived by Equation (10):

$$
\left\{\begin{array}{l}
d_{C 1 W}^{s}=\beta_{12} \cdot D C B_{C 1 W-C 2 W}^{s} \\
d_{C 2 W}^{s}=-\alpha_{12} \cdot D C B_{C 1 W-C 2 W}^{s}
\end{array}\right.
$$

The other type code biases such as C1C, C5Q and C5X can be easily derived by Equation (12):

$$
\left\{\begin{array}{l}
d_{C 1 C}^{s}=D C B_{C 1 C-C 1 W}^{s}+\beta_{12} \cdot D C B_{C 1 W-C 2 W}^{s} \\
d_{C 5 Q}^{s}=D C B_{C 1 C-C 1 W}^{s}+\beta_{12}^{s} \cdot D C B_{C 1 W-C 2 W}^{s}-D C B_{C 1 C-C 5 Q}^{s} \\
d_{C 5 X}^{s}=D C B_{C 1 C-C 1 W}^{s}+\beta_{12} \cdot D C B_{C 1 W-C 2 W}^{s}-D C B_{C 1 C-C 5 X}^{s}
\end{array}\right.
$$


Considering Equations (3), (12) and (13), the satellite code bias correction for GPS triple-frequency pseudo-range observations can be conveniently expressed as:

$$
\left\{\begin{array}{l}
\widetilde{P}_{r, \mathrm{C} 1 W}^{s}=P_{r, \mathrm{C} 1 W}^{s}+d_{\mathrm{C} 1 W}^{s} \\
\widetilde{P}_{r, \mathrm{C} 2 W}=P_{r, \mathrm{C} 2 W}^{s}+d_{\mathrm{C} 2 W}^{s} \\
\widetilde{P}_{r, \mathrm{C} 5 \mathrm{X}}^{s}=P_{r, \mathrm{C} 5 \mathrm{X}}^{s}+d_{\mathrm{C} 5 \mathrm{X}}^{s}
\end{array}\right.
$$

where $\widetilde{P}_{r, \mathrm{C} 1 W}^{s}, \widetilde{P}_{r, \mathrm{C} 2 W}^{s}$ and $\widetilde{P}_{r, \mathrm{C} 5 \mathrm{X}}^{s}$ denote the pseudo-range observations where the code biases have been corrected. It should be noted that only a relative relation between satellite code biases is corrected to pseudo-range observations, therefore $d_{C 1 W^{\prime}}^{s} d_{C 2 W}^{s}$ and $d_{C 5 X}^{s}$ should be interpreted as pseudo-absolute code biases. In fact, these pseudo-absolute code biases can be considered as observable-specific SCB which can be directly corrected to the original pseudo-range observations.

\subsection{Observable-Specific Satellite Phase Biases}

Time-invariant satellite phase bias correction, also called SPB correction, which aims to recover the integer property of carrier phase ambiguity, is the prerequisite of conducting PPP AR. The traditional method of SPB correction, which commonly applies the corresponding SPB products to correct float ambiguities, can be expressed as [4,6,9]:

$$
\Delta \bar{N}_{r}^{s}=\Delta \widehat{N}_{r}^{s}+\Delta \bar{b}^{s}
$$

where $\Delta \hat{N}_{r}^{s}$ denote SD float ambiguity estimates (cycles); $\Delta \bar{b}^{s}$ denote SD satellite SPB (cycles); $\Delta \bar{N}_{r}^{s}$ denote SD float ambiguity estimates which have been corrected with SPB products. As can be seen, the traditional method of SPB correction depends on the form of float ambiguity. For example, if float ambiguity is the wide-lane (WL) form, the SPB product should correspond to WL SPB; if the float ambiguity is the UDUC form, the SPB product should correspond to UDUC SPB. Similar to the observable-specific SCB, an alternative SPB correction method for UDUC-PPP can be described as below $[5,27]$ :

$$
\left\{\begin{array}{l}
\bar{L}_{r, 1}^{s}=L_{r, 1}^{s}+\bar{b}_{1}^{s} \\
\bar{L}_{r, 2}^{s}=L_{r, 2}^{s}+\bar{b}_{2}^{s} \\
\bar{L}_{r, 5}^{s}=L_{r, 5}^{s}+\bar{b}_{5}^{s}
\end{array}\right.
$$

where $\bar{b}_{1}, \bar{b}_{2}^{s}$ and $\bar{b}_{5}^{\mathcal{s}}$ are UDUC SPB products for $L_{r, 1}^{s}, L_{r, 2}^{s}$ and $L_{r, 5}^{s}$ original phase observations, respectively (m); and $\bar{L}_{r, 1}^{S}, \bar{L}_{r, 2}^{S}$ and $\bar{L}_{r, 5}^{S}$ are carrier-phase observations without time-invariant SPB. The UDUC SPB can also be considered as observable-specific SPB which can be directly corrected to original phase observations on each frequency. It is obvious that the observable-specific SPB can be easily and conveniently extended for multi-frequency and multi-GNSS PPP AR and also applied to different PPP models.

According to Equations (7)-(9), the time-variant SPBs are contained in both pseudo-range and carrier-phase observations. The time-variant SPBs which are contained in pseudo-range observations can be ignored because their effects are usually smaller than pseudo-range noise [23]. Therefore, handling the time-variant satellite phase bias for triple-frequency UDUC-PPP mainly aims to correct the IFCB on L5 carrier-phase observations. According to Equations (8) and (9), the observable-specific IFCB on L5 frequency can be straightforwardly formulated as:

$$
I F C B^{s}=\alpha_{12} \cdot\left(1-\gamma_{5} / \gamma_{2}\right) \cdot \Delta b_{1}^{s}-\beta_{12} \cdot\left(\gamma_{5}-1\right) \cdot \Delta b_{2}^{s}-\Delta b_{5}^{s}
$$

As can be seen, observable-specific IFCB is a linear combination of time-variant satellite phase bias on L1, L2 and L5 frequencies. Similar to the observable-specific SPB, it can be directly corrected to L5 
phase observations at the user end after being estimated by PPP network solutions or other strategies, e.g., [17], or [23] or [28]. Thus, the observable-specific IFCB correction can be easily expressed as:

$$
\widetilde{L}_{r, 5}^{s}=L_{r, 5}^{s}+I F C B^{s}
$$

where $\widetilde{L}_{r, 5}^{s}$ denotes the $\mathrm{L} 5$ phase observations without $I F C B^{s}$.

\subsection{Observable-Specific Satellite Bias Corrections for UDUC-PPP AR}

The process of observable-specific bias corrections for triple-frequency UDUC-PPP AR is shown in Figure 1. As can be seen, it includes the formation of observable-specific satellite code and phase biases at the service end as well as observable-specific bias corrections for multi-frequency UDUC-PPP AR at the user end. At the service end, firstly we need to estimate observable-specific IFCB using triple-frequency GPS observations. Observable-specific IFCB can be obtained by converting ionosphere-free IFCB, which is commonly estimated by differencing ionosphere-free combinations of L1/L2 and L1/L5 phase observations [23]. Then, we convert the DCB product (e.g., DLR DCB product, estimated using the GIM model and differenced intra-/inter-frequency pseudo-range observations [11]) to an observable-specific SCB product. Next, observable-specific SPB can be estimated with consideration of observable-specific IFCB and SCB corrections. Finally, we need to combine observable-specific IFCB, SPB and SCB to generate the file (SINEX_BIAS) for observable-specific bias corrections at the PPP user end. A detailed description of the SINEX_BIAS file is given in "Appendix A." At the user end, we can innovate to directly apply the SINEX_BIAS file to correct the original pseudo-range and carrier-phase observations. Then, float ambiguities with an integer property and corresponding variance-covariance matrix can be obtained when conducting float UDUC-PPP. Next, UDUC integer ambiguities can be searched using the LAMBDA method [29,30]. Eventually, the UDUC integer ambiguities can be applied as constraint conditions to get the ambiguity-fixed UDUC-PPP [6].

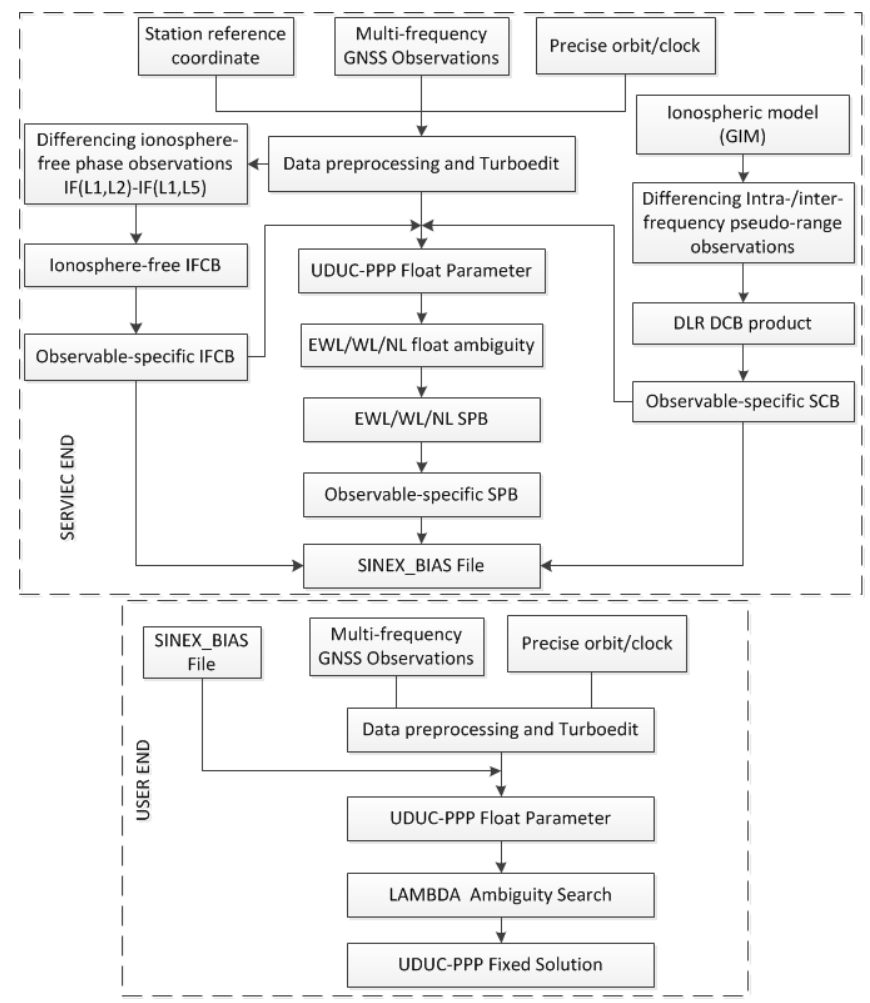

Figure 1. Flowchart of formation of observable-specific satellite code and phase biases at the service end and observable-specific bias corrections for multi-frequency UDUC-PPP AR at the user end. 


\section{Experiment and Results}

In this section, we investigate the performance of triple-frequency GPS UDCU-PPP AR with observable-specific satellite code and phase biases. We start with introducing the generation of SINEX_BIAS files using real triple-frequency GPS observation data, as well as triple-frequency GPS UDUC-PPP AR strategies at the user end. Next, we respectively study the performance of triple-frequency GPS UDUC-PPP AR with observable-specific SCB and IFCB in terms of time-to-first-fix (TTFF) and positioning accuracy.

\subsection{Data and UDUC-PPP AR Strategy}

As shown in Figure 2, 142 MGEX stations, which can track GPS triple-frequency signals on day 92-99 of 2017, are used to estimate triple-frequency observable-specific SPB as well as observable-specific IFCB for L5 carrier-phase observations. Observable-specific SCB are converted from the DCB products released by DLR. A SINEX_BIAS file could be generated by combining SCB products as well as IFCB and SPB estimates. At the user ends, the performance of triple-frequency GPS UDUC-PPP AR using a SINEX_BIAS file is investigated at twelve stations, which are shown in Table 2. Table 2 shows the receiver type of the station, the number of visible triple-frequency GPS satellites per epoch, the number of visible GPS satellites per epoch, the total number of available GPS satellites and the available triple-frequency GPS satellites at each station. As can be seen, currently about 5-6 triple-frequency GPS satellites per epoch can be used for UDUC-PPP AR.

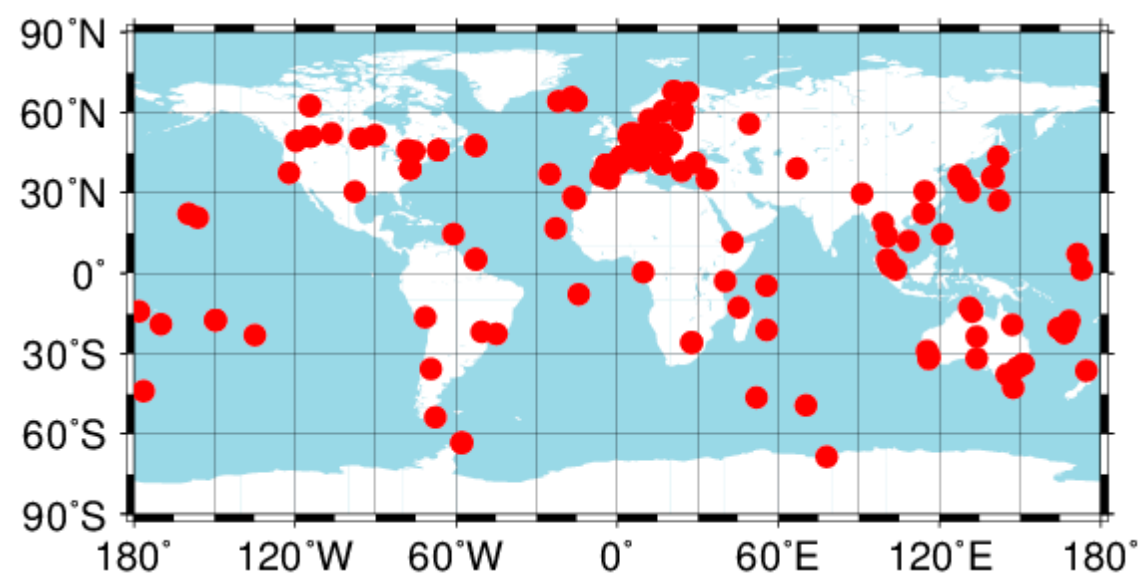

Figure 2. Distribution of selected stations with GPS triple-frequency observations.

Table 2. Information of the twelve user stations including receiver type, the number of visible triple-frequency GPS satellites per epoch (Tri Sat Num), the number of visible GPS satellites per epoch (Sat Num), the total number of available GPS satellites and available triple-frequency GPS satellites (Total (Tri) Sat Num).

\begin{tabular}{ccccc}
\hline Station & Receiver Type & $\begin{array}{c}\text { Tri Sat Num } \\
\text { (Epoch) }\end{array}$ & $\begin{array}{c}\text { Sat Num } \\
\text { (Epoch) }\end{array}$ & $\begin{array}{c}\text { Total (Tri) Sat Num } \\
\text { (Available) }\end{array}$ \\
\hline AJAC & LEICA GR25 & $5-6$ & $9-11$ & $31(12)$ \\
BOR1 & TRIMBLE NETR9 & $5-7$ & $10-13$ & $31(12)$ \\
BRUX & SEPT POLARX4TR & $5-7$ & $12-13$ & $31(12)$ \\
GOP6 & LEICA GRX1200+GNSS & $5-6$ & $8-12$ & $31(12)$ \\
GRAC & LEICA GR25 & $5-6$ & $9-12$ & $31(12)$ \\
KOS1 & SEPT POLARX4 & $5-7$ & $10-13$ & $31(12)$ \\
MATG & LEICA GRX1200+GNSS & $5-6$ & $9-16$ & $31(12)$ \\
TLSG & SEPT POLARX4TR & $5-6$ & $9-14$ & $31(12)$ \\
WARN & JAVAD TRE_G3TH & $5-7$ & $10-14$ & $31(12)$ \\
WTZR & LEICA GR25 & $5-6$ & $9-12$ & $31(12)$ \\
WTZS & SEPT POLARX4TR & $5-6$ & $9-12$ & $31(12)$ \\
WTZZ & TRE_G3TH & $5-6$ & $9-12$ & $31(12)$ \\
\hline
\end{tabular}


As a comparison, weekly coordinate solutions in SINEX format are considered as the reference coordinates. IGS precise orbit and clock products at intervals of $5 \mathrm{~min}$ and $30 \mathrm{~s}$ are used, respectively. Carrier-phase observations are given a standard deviation of 3 millimeters, while code observations are de-weighted by a factor of 100. More detailed strategies about GPS UDUC-PPP AR are described in Table 3.

Table 3. GPS un-differenced and uncombined precise point positioning ambiguity resolution (UDUC-PPP AR) processing strategy.

\begin{tabular}{|c|c|}
\hline Item & Strategy \\
\hline Estimator & Sequential least square estimator \\
\hline Observations & Original carrier-phase and pseudo-range observations \\
\hline Signal selection & GPS: L1/L2/L5 \\
\hline Satellite selection & Block IIF satellite with triple-frequency signals \\
\hline Sampling rate & $30 \mathrm{~s}$ \\
\hline Elevation cutoff & $15^{\circ}$ \\
\hline Observations weight & Elevation-dependent weight \\
\hline Ionospheric delay & Estimated as random walk process \\
\hline Tropospheric delay & $\begin{array}{l}\text { Dry component: corrected with the Saastamoinen model } \\
\text { Wet component: estimated as random-walk process, GMF mapping function }\end{array}$ \\
\hline Receiver clock & Estimated as white noise \\
\hline Station displacement & $\begin{array}{l}\text { Corrected by IERS Convention 2010, including Solid Earth tide, } \\
\text { pole tide and ocean tide loading [31] }\end{array}$ \\
\hline Satellite PCO/PCV & Corrected using IGS14 ANTEX file \\
\hline Phase-windup effect & Corrected [32] \\
\hline Relativistic effect & Applied \\
\hline Station coordinate & Static: estimated as constants, kinematic: estimated as white noise \\
\hline Observable-specific SCB & Type: $\mathrm{C} 1 \mathrm{C}$ or $\mathrm{C} 1 \mathrm{~W} / \mathrm{C} 2 \mathrm{~W} / \mathrm{C} 5 \mathrm{X}$ \\
\hline Observable-specific SPB & Type: L1C or L1W/L2W/L5X \\
\hline Observable-specific IFCB & Type: L5X \\
\hline
\end{tabular}

\subsection{Performance of UDUC-PPP AR with Observable-Specific SCB}

As a typical example, Figure 3 shows the performance of static and kinematic triple-frequency UDUC-PPP AR with or without observable-specific SCB corrections during the initialization, respectively. As can be seen, both static and kinematic UDUC-PPP AR with SCB corrections reduced TTFF, compared with that without SCB corrections. Static UDUC-PPP AR without SCB corrections took $18.5 \mathrm{~min}$ to finish the first fix while static UDUC-PPP AR with SCB corrections only took $15.5 \mathrm{~min}$, with TTFF reduced by $16.2 \%$; kinematic UDUC-PPP AR without SCB corrections took $31.5 \mathrm{~min}$ to finish the first fix, while kinematic UDUC-PPP AR with SCB corrections only took $15 \mathrm{~min}$, with TTFF reduced by $52.3 \%$. Compared with static UDUC-PPP AR, SCB had more significant influence on kinematic UDUC-PPP AR, due to the fact that the static solutions using multi-epoch observations can easily smooth $\mathrm{SCB}$, which was contained in a pseudo-range measurement, while the kinematic solutions using a single-epoch observation are more sensitive to SCB contained in a pseudo-range measurement. However, SCB could hardly influence the positioning accuracy of static or kinematic UDUC-PPP AR when ambiguities were fixed. The main reason is that the pseudo-range measurements with relatively low weight ratio have little influence on the positioning accuracy. Therefore, SCB mainly influence the TTFF of kinematic UDUC-PPP AR. 

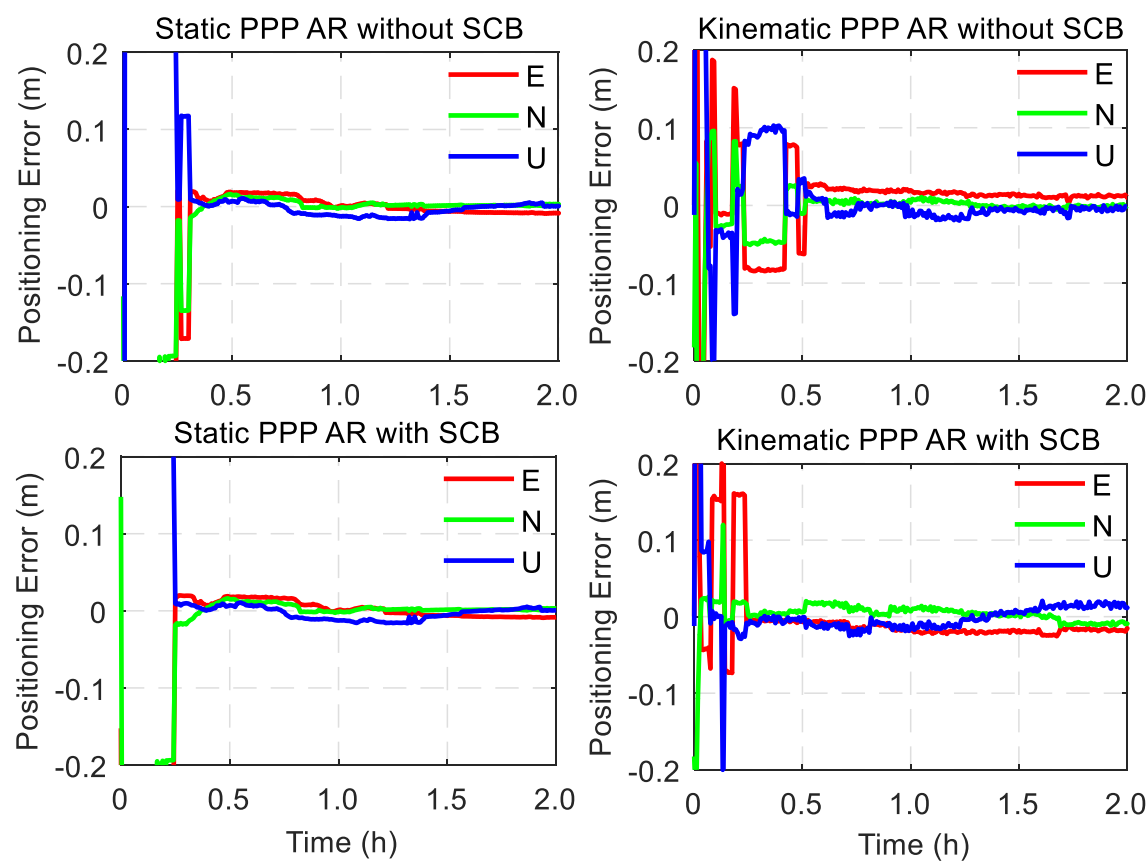

Figure 3. Two-h time series of position errors for triple-frequency UDUC-PPP AR with or without observable-specific SCB corrections at BRUX stations.

In order to further study the performance of kinematic UDUC-PPP AR with observable-specific SCB corrections, the TTFF of kinematic UDUC-PPP AR with or without SCB corrections at the ten different user stations are shown in Figure 4. The averaged TTFF for two groups of solutions are also shown in Figure 4. As can be seen, the effects of SCB corrections on UDUC-PPP AR at each station are different so that the improvement of TTFF varied at different stations. For example, the improvement of TTFF at BRUX station was much more than that at GOP6 station. This variation depends on many factors and one of the factors we guess depends on the quality of pseudo-range observations at different user stations. If the accuracy of pseudo-range observations at one test station is relatively high, it will become higher when the SCBs are corrected, thus leading to much improvement of the TTFF. On the contrary, if the quality of pseudo-range observations is poor, it will not become better, even if the SCBs are corrected, and the TTFF cannot be improved too much. On average, the statistical results of the ten stations demonstrated that the TTFF of kinematic UDUC-PPP AR with SCB corrections was about $22 \mathrm{~min}$, which reduced the TTFF by $29 \%$, compared to that without SCB corrections, which was about $31 \mathrm{~min}$.

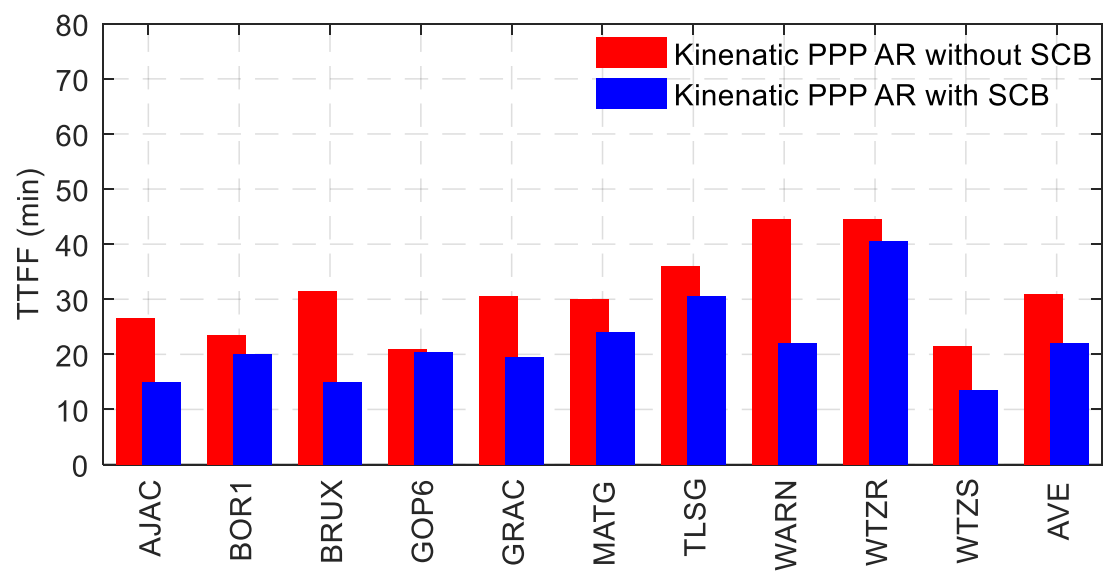

Figure 4. Time-to-first-fix (TTFF) of kinematic UDUC-PPP AR with or without observable-specific satellite code bias (SCB) corrections at ten stations. 


\subsection{Performance of UDUC-PPP AR with Observable-Specific IFCB}

Figure 5 shows a typical 3-h time series of position errors for triple-frequency static float UDUC-PPP as well as UDUC-PPP AR with observable-specific IFCB corrections at BRUX stations. It is pretty obvious that the performance of both float UDUC-PPP and UDUC-PPP AR with IFCB corrections could be significantly improved in terms of convergence time and positioning accuracy, compared with that without IFCB corrections. The TTFF of UDUC-PPP AR without IFCB corrections was about $68.5 \mathrm{~min}$ while the TTFF of UDUC-PPP AR with IFCB is about $15.5 \mathrm{~min}$, with about $77 \%$ improvement at this station. With about 3-h observations, the positioning accuracy of UDUC-PPP AR without IFCB was about $2.6 \mathrm{~cm}$ and $2.3 \mathrm{~cm}$ in a horizontal and vertical direction, respectively, while the positioning accuracy of the UDUC-PPP AR without IFCB could achieve about $0.7 \mathrm{~cm}$ and $1.8 \mathrm{~cm}$ in comparison, with an improvement of about $73 \%$ and $22 \%$ in a horizontal and vertical direction, respectively. Furthermore, it should be noted that the effects of IFCB corrections on float UDUC-PPP and UDUC-PPP AR were distinct. The positioning accuracy of float solutions without IFCB corrections can be improved with the float parameters being smoothed over time. In contrast, the positioning accuracy of fixed solutions without IFCB corrections at 2.2-2.3 $\mathrm{h}$ was even worse than that at 1.2-1.4 $\mathrm{h}$. This is mainly because IFCB presented continuous periodic variation, as shown in Figure 6, thus leading to the number of incorrect fixed ambiguities also changing. When the number of incorrect fixed ambiguities becomes large, the positioning accuracy will immediately become relatively poor, even if UDUC-PPP AR had finished the first fix. Therefore, it is an essential prerequisite to correct IFCB before conducting triple-frequency UDUC-PPP AR.
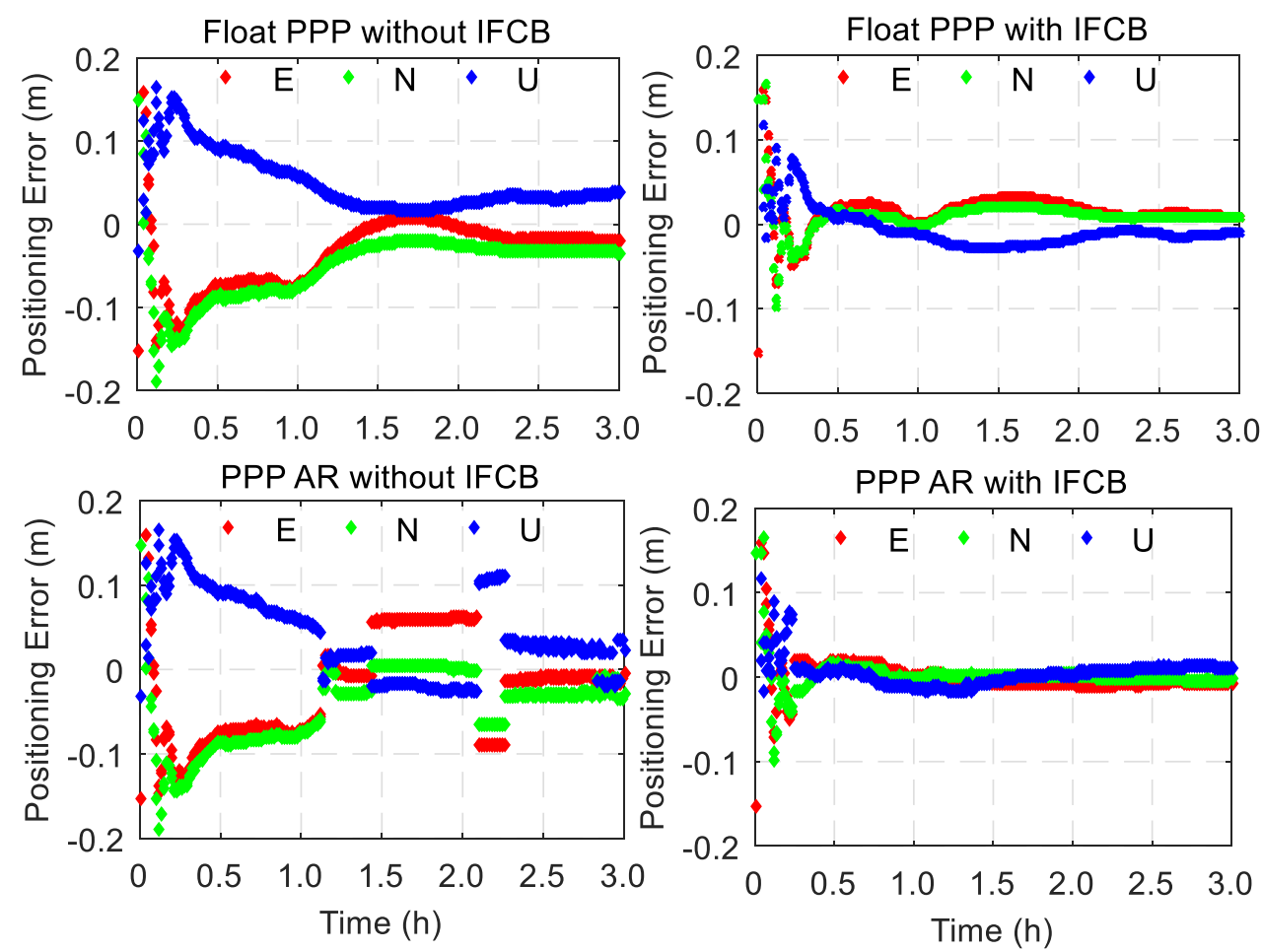

Figure 5. Three-h time series of position errors for triple-frequency static float PPP and PPP AR with or without observable-specific IFCB corrections at BRUX stations. 


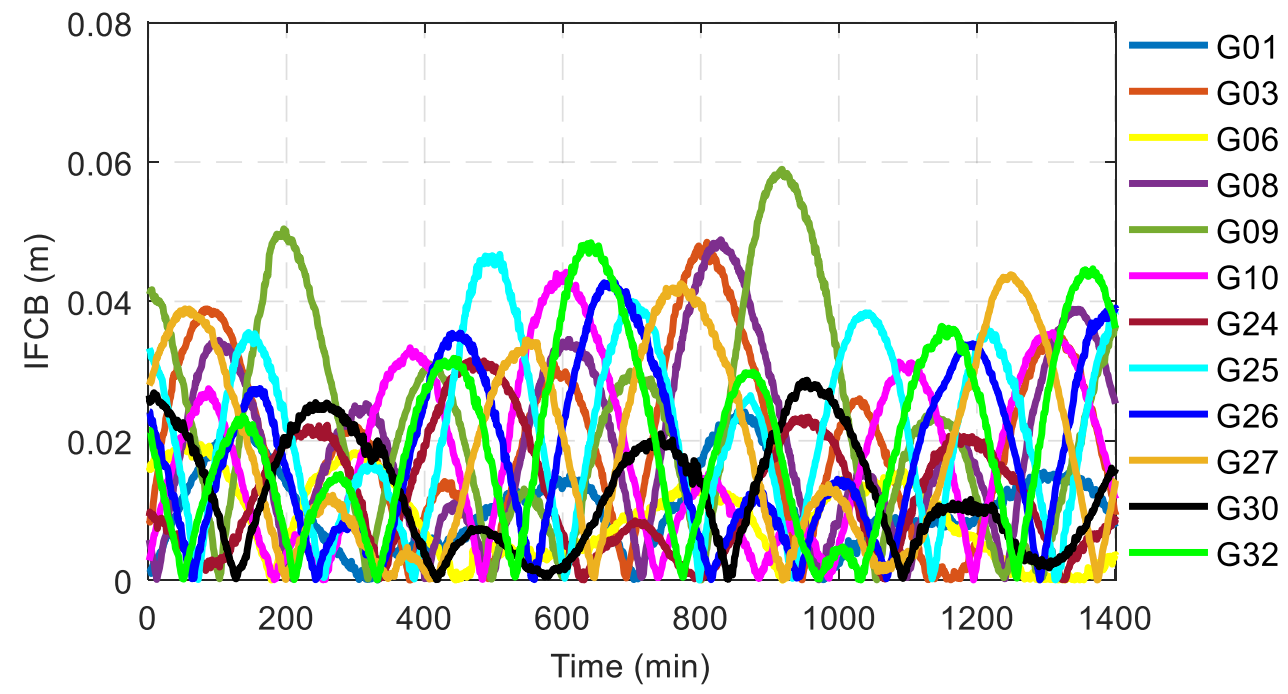

Figure 6. Time series of observable-specific IFCB estimates for Block IIF satellites on DOY 092, 2017.

For the purpose of further studying the TTFF of triple-frequency GPS UDUC-PPP AR with observable-specific IFCB corrections, we conducted a statistical analysis of the TTFF of static UDUC-PPP AR with or without IFCB corrections at twelve different user stations, as shown in Figure 7. The averaged TTFF for the two groups of solutions are also shown in Figure 7. It can be demonstrated that the TTFF of UDUC-PPP AR with IFCB corrections is much shorter than that without IFCB corrections at all user stations. The averaged TTFF of UDUC-PPP AR without IFCB corrections was about $43.8 \mathrm{~min}$, while the averaged TTFF of UDUC-PPP AR with IFCB corrections was about $15.6 \mathrm{~min}$, with about $64.3 \%$ improvement. Compared with Figure 4 above, it is obvious that the effect of IFCB on TTFF was more significant than that of SCB on TTFF. This is because IFCB undermined carrier-phase measurements while SCB undermined pseudo-range measurements, and the destruction of phase observations would undoubtedly have a more disastrous impact on integer ambiguity resolution. In addition, the effects of IFCB on TTFF were distinct at different station, which was mainly due to the fact that there were different IFCB of different satellites observed at each station, leading to different effects on PPP AR.

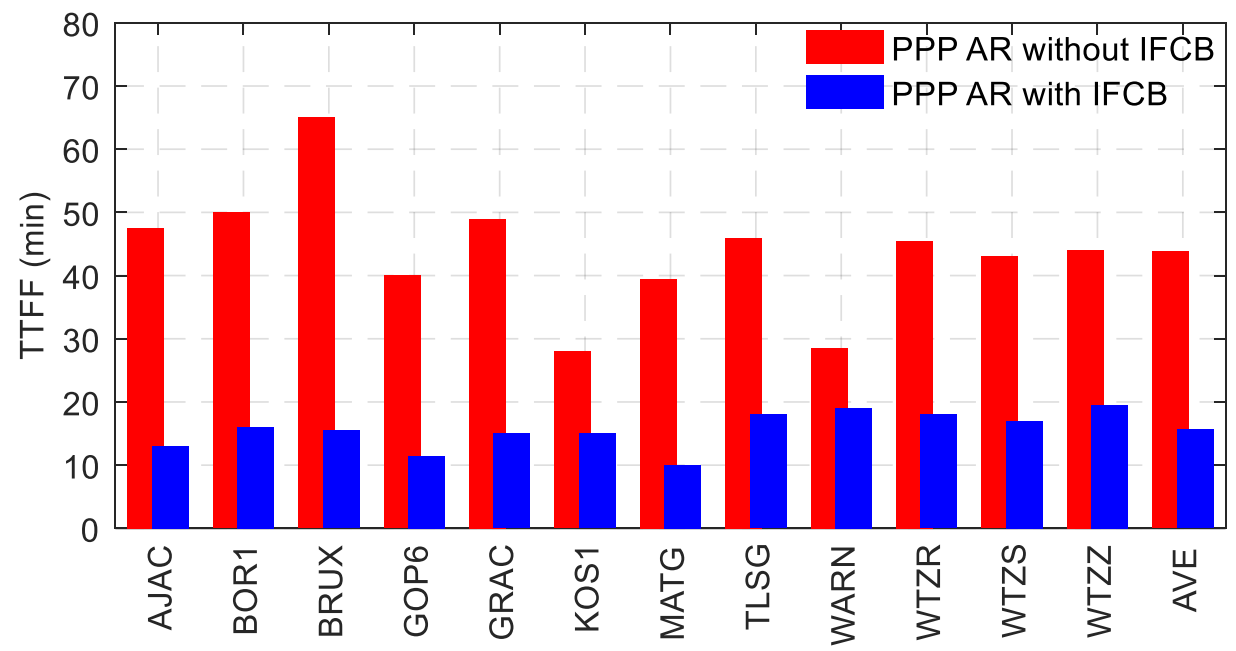

Figure 7. TTFF of static UDUC-PPP AR with or without observable-specific IFCB corrections at twelve different user stations.

In order to further verify the positioning accuracy of triple-frequency GPS UDUC-PPP AR with observable-specific IFCB corrections, we conducted a statistical analysis of the root mean square (RMS) of positioning errors of the above user stations at different sessions in the east, north and up directions 
for UDUC-PPP AR with or without IFCB, as shown in Figure 8. It could be seen that the positioning accuracy of UDUC-PPP AR with IFCB corrections was obviously much higher at any session, compared with that without IFCB corrections. The results showed that the positioning accuracy of UDUC-PPP AR with IFCB corrections at TTFF could reach $2.5 \mathrm{~cm}, 2.3 \mathrm{~cm}$ and $2.8 \mathrm{~cm}$ in the east, north and up directions respectively, with improvements of $36 \%, 34 \%$ and $29 \%$ in three directions, compared with that without IFCB corrections; with 3-h observation, the positioning accuracy with IFCB corrections could reach about $1.3 \mathrm{~cm}, 1.1 \mathrm{~cm}$ and $1.6 \mathrm{~cm}$ in three directions respectively, with improvements of $42 \%, 58 \%$ and $56 \%$ in three directions, compared with that without IFCB corrections. Furthermore, it should be mentioned that the positioning accuracy of UDUC-PPP AR without IFCB corrections at 1.5 $\mathrm{h}$ was even worse than that at TTFF. The reason may be that the periodical IFCB values at $1.5 \mathrm{~h}$ were larger, thus leading to a greater impact on AR, which was also similar to the discussions above.
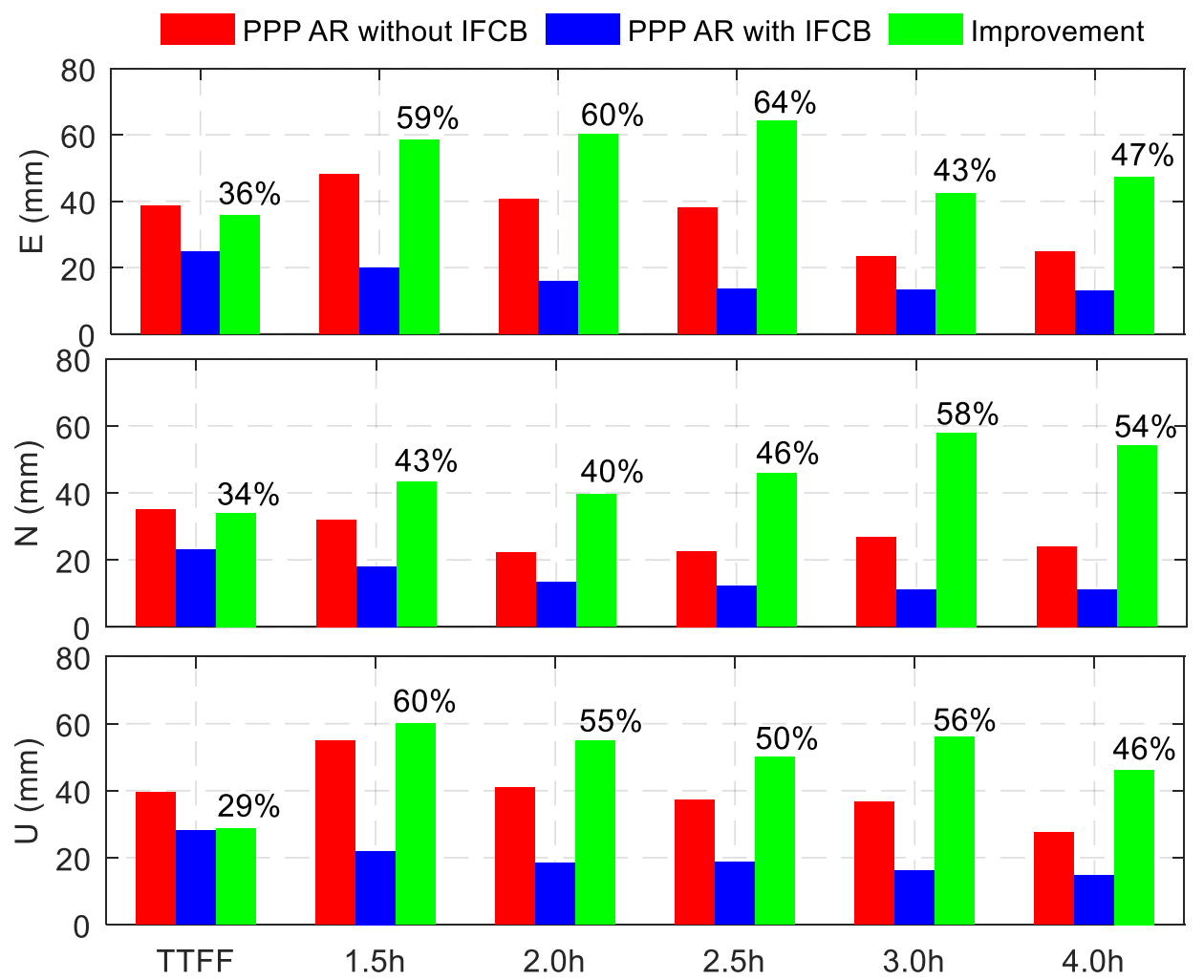

Figure 8. Root mean square (RMS) of positioning errors at different sessions in the east, north and up components for UDUC-PPP AR with or without IFCB corrections.

\section{Discussion}

With the emergence of multi-GNSS and multi-frequency signals, the treatment of satellite code and phase biases is essential for PPP AR. Compared with the traditional approach, the presented OSB correction method, which includes observable-specific SCB and SPB as well as IFCB correction, is more well suited for multi-GNSS and multi-frequency PPP AR. This is mainly because the OSB correction method can directly correct kinds of observable-specific satellite biases to GNSS original observations, without considering the correlation between different frequency signals and even different PPP AR model. Based on the OSB correction method, the study about the performance of UDUC-PPP AR using real triple-frequency GPS observation data is another new contribution. According to the experiment results above, it has been shown that triple-frequency UDUC-PPP with observable-specific SPB can achieve ambiguity-fixed UDUC-PPP, triple-frequency UDUC-PPP AR with observable-specific SCB can improve the TTFF of kinematic ambiguity-fixed solutions and triple-frequency UDUC-PPP AR with observable-specific IFCB can improve both the TTFF and positioning accuracy of ambiguity-fixed 
solutions. The conclusions are as expected and the results are easily explained, which was discussed in Section 3. In addition, it should be mentioned that the TTFF of triple-frequency GPS static UDUC-PPP AR is 15.6 min. Comparing with previous studies, as shown in Table 4, the dual-frequency GPS static PPP AR is about $20 \mathrm{~min}$ [34,34], and it can be found that athough the number of available triple-frequency GPS satellites is less than the number of available dual-frequency GPS satellites, the TTFF of triple-frequency PPP AR is still faster than that of dual-frequency PPP AR, indicating that triple-frequency observations are quite helpful for PPP rapid convergence. At the same time, the TTFF of triple-frequency BDS UDUC-PPP AR and triple-frequency Galileo UDUC-PPP AR is also faster than that of dual-frequency BDS UDUC-PPP AR and dual-frequency Galileo UDUC-PPP AR $[4,6]$, which further verifies the fact that triple-frequency observations of other GNSS constellations can also improve the performance of PPP AR. As can be seen from Table 4, although the TTFF of triple-frequency PPP AR has been significantly improved compared with dual-frequency PPP AR, it is still long. One of the reasons is that the number of available triple-frequency satellites in a single GNSS constellation is still limited. In order to increase the number of triple-frequency satellites, we will conduct research on multi-GNSS and multi-frequency PPP AR next.

Table 4. Performance comparison of dual-frequency and triple-frequency PPP AR for BDS-2, Galileo and GPS.

\begin{tabular}{lcccc}
\hline Item/Reference & TTFF (min) & $\begin{array}{c}\text { Sat Num } \\
\text { (Epoch) }\end{array}$ & Data & $\begin{array}{c}\text { Signal } \\
\text { Selection }\end{array}$ \\
\hline Dual-frequency BDS-2 PPP AR/[4] & 31 & $6-7$ & IGS MGEX & B1/B2 \\
Triple-frequency BDS-2 PPP AR/[4] & 27.9 & $6-7$ & IGS MGEX & B1/B2/B3 \\
Dual-frequency Galileo PPP AR/ [6] & 20 & $5-8$ & IGS MGEX & E1/E5a \\
Triple-frequency Galileo PPP AR/[6] & 16 & $5-8$ & IGS MGEX & E1/E5a/E5b \\
Dual-frequency GPS PPP AR/[33]/[34] & $22.6 / 20.8$ & $9-14$ & IGS MEGX & L1/L2 \\
Triple-frequency GPS PPP AR & 15.6 & $5-7$ & IGS MEGX & L1/L2/L5 \\
\hline
\end{tabular}

\section{Conclusions}

Multi-frequency signals have been demonstrated to bring significant improvement to the performance of PPP AR. In order to conveniently conduct multi-frequency and also multi-GNSS UDUC-PPP AR, we proposed a method to uniformly correct time-invariant and time-variant satellite biases and also designed a corresponding file format of bias corrections. In addition, the effects of SCB and IFCB on the performance of triple-frequency UDUC-PPP AR are evaluated, in terms of TTFF and positioning accuracy.

We took advantage of 142 MGEX stations to estimate the SPB and IFCB to generate a SINEX_BIAS file at the service end and also verified the effects of SCB and IFCB on the performance of GPS triple-frequency UDUC-PPP AR at the user end, in terms of TTFF as well as positioning accuracy. It was concluded that the effects of SCB corrections on kinematic UDUC-PPP AR was more significant, compared with that on static UDUC-PPP AR; SCB mainly influenced TTFF instead of positioning accuracy. The results demonstrated that the averaged TTFF of kinematic UDUC-PPP AR with SCB corrections could reach 22 min, with about 29\% improvement, compared with that without SCB corrections. IFCB could influence both TTFF and positioning accuracy and the effects could be constantly changing, due to periodic variation of IFCB estimates. It was shown that the averaged TTFF of static UDUC-PPP AR with IFCB corrections was about $15.6 \mathrm{~min}$, with about $64.3 \%$ improvement, compared with that without IFCB corrections; the positioning accuracy of UDUC-PPP AR with IFCB corrections at TTFF could reach $2.5 \mathrm{~cm}, 2.3 \mathrm{~cm}$ and $2.8 \mathrm{~cm}$ in the east, north and up directions, respectively, with improvements of $36 \%, 34 \%$ and $29 \%$ in three directions, compared with that without IFCB corrections.

It is worth mentioning that the TTFF of GPS triple-frequency UDUC-PPP AR is still relative long. One of reasons we think this may be due to the fact that only a small number of the GPS satellite transmitting triple-frequency signals can be observed at one station. Therefore, we plan to further 
study the performance of UDUC-PPP AR with triple-frequency GPS, BDS and Galileo observations. In addition, the TTFF of triple-frequency UDUC-PPP AR at different stations is obviously distinct, which also needs to be studied further.

Author Contributions: Funding acquisition, F.G.; methodology, G.L. and F.G.; software, G.L.; supervision, J.W. and M.D.; validation, G.L.; writing-original draft, G.L.; writing-review and editing, G.L. and L.Q. All authors have read and agreed to the published version of the manuscript.

Funding: This research was funded by the National Natural Science Foundation of China (No. 41774034), National Natural Science Foundation of China (No. 41874029), Beijing Natural Science Foundation (4204094), General Science and Research Project for Science and Technology Program of Beijing Education Commission (KM201910016007) and Fundamental Research Funds for Beijing University of Civil Engineering and Architecture (No. X18007).

Acknowledgments: The authors are grateful to the many individuals and organizations worldwide who contribute to the International GNSS Service. We also gratefully acknowledge the use of Generic Mapping Tool (GMT) software.

Conflicts of Interest: The authors declare no conflicts of interest.

\section{Appendix A Basic Format of SINEX_BIAS File for UDUC-PPP AR}

In order to facilitate unified processing of satellite code and phase bias for multi-frequency and multi-GNSS UDUC-PPP AR, we designed a basic file format of unified bias correction, with reference to a SINEX_BIAS file [14]. The basic format of SINEX_BIAS files for triple-frequency GPS UDUC-PPP AR is shown in Figure A1. Taking the G01 satellite as an example, OSB_SCB, OSB_SPB and OSB_IFCB in the "*BIAS" column of the SINEX_BIAS file represent SCB for each pseudo-range type, SPB for each carrier-phase type and IFCB for L5X observation, respectively. It is obvious that the form of these biases is observable-specific signal bias (OSB), which can be directly corrected to corresponding original pseudo-range and carrier-phase observations. The time difference between "BIAS_START" and "BIAS_END" denotes the $30 \mathrm{~s}$ sampling interval for these biases. "UNIT" denotes the unit of these biases. "_ESTIMATED_VALUE_" and "STD_DEV" denote the bias estimates and corresponding standard deviation.

\begin{tabular}{|c|c|c|c|c|c|c|c|c|}
\hline \multicolumn{9}{|c|}{ +BIAS/SOLUTION } \\
\hline *BIAS & SVN_ & PRN & OBS & BIAS_START & BIAS_END_ & UNIT & ESTIMATED_VALUE & STD_DEV \\
\hline OSB_SCB & $\mathrm{G} 06 \overline{3}$ & G01 & $\mathrm{C} 1 \mathrm{C}$ & $2017 \overline{0} 092: 0 \overline{0000}$ & $2017: 092 \overline{00030}$ & ns & $1 . \overline{6132}$ & $0.0 \overline{081}$ \\
\hline OSB_SCB & G063 & G01 & $\mathrm{C} 1 \mathrm{~W}$ & 2017:092:00000 & $2017: 092: 00030$ & ns & -1.1810 & 0.0090 \\
\hline OSB_SCB & G063 & G01 & $\mathrm{C} 2 \mathrm{~W}$ & 2017:092:00000 & $2017: 092: 00030$ & ns & -8.9380 & 0.0215 \\
\hline OSB_SCB & G063 & G01 & $\mathrm{C} 2 \mathrm{~S}$ & 2017:092:00000 & $2017: 092: 00030$ & ns & 1.4530 & 0.0135 \\
\hline OSB_SCB & G063 & G01 & $\mathrm{C} 2 \mathrm{~L}$ & 2017:092:00000 & $2017: 092: 00030$ & ns & 1.3140 & 0.0070 \\
\hline OSB_SCB & G063 & G01 & $\mathrm{C} 5 \mathrm{Q}$ & 2017:092:00000 & $2017: 092: 00030$ & ns & 0.6380 & 0.0515 \\
\hline OSB_SCB & G063 & G01 & C5X & 2017:092:00000 & $2017: 092: 00030$ & ns & 1.0580 & 0.0180 \\
\hline OSB_SPB & G063 & G01 & L1C & 2017:092:00000 & $2017: 092: 00030$ & cyc & 0.4490 & 0.0130 \\
\hline OSB_SPB & G063 & G01 & $\mathrm{L} 2 \mathrm{~W}$ & 2017:092:00000 & $2017: 092: 00030$ & cyc & -0.2330 & 0.0190 \\
\hline OSB_SPB & G063 & G01 & L2X & 2017:092:00000 & $2017: 092: 00030$ & cyc & -0.1330 & 0.0260 \\
\hline OSB_SPB & G063 & G01 & L5X & 2017:092:00000 & $2017: 092: 00030$ & cyc & 0.2900 & 0.0160 \\
\hline OSB IFCB & G063 & G01 & L5X & 2017:092:00000 & $2017: 092: 00030$ & $\mathrm{~m}$ & -0.0013 & 0.0008 \\
\hline
\end{tabular}

Figure A1. RMS of positioning errors at different sessions in the east, north and up components for UDUC-PPP AR with or without IFCB corrections.

\section{References}

1. Zrinjski, M.; Barković, Đ.; Matika, K. Razvoj i modernizacija GNSS-a. Geodetski. List. 2019, 73, 45-65.

2. Geng, J.; Bock, Y. Triple-frequency GPS precise point positioning with rapid ambiguity resolution. J. Geod. 2013, 87, 449-460. [CrossRef]

3. Gu, S.; Lou, Y.; Shi, C.; Liu, J. BeiDou phase bias estimation and its application in precise point positioning with triple-frequency observable. J. Geod. 2015, 89, 979-992. [CrossRef] 
4. Li, P.; Zhang, X.; Ge, M.; Schuh, H. Three-frequency BDS precise point positioning ambiguity resolution based on raw observables. J. Geod. 2018, 92, 1357-1369. [CrossRef]

5. Innovation: Instantaneous Centimeter-Level Multi-Frequency Precise Point Positioning. Available online: https://www.gpsworld.com/innovation-instantaneous-centimeter-level-multi-frequency-precisepoint-positioning/ (accessed on 10 July 2020).

6. Liu, G.; Zhang, X.; Li, P. Improving the performance of Galileo uncombined precise point positioning ambiguity resolution using triple-frequency observations. Remote Sens. 2019, 11, 341. [CrossRef]

7. Li, X.; Li, X.; Liu, G.; Xie, W.; Guo, F.; Yuan, Y.; Zhang, K.; Feng, G. Triple-frequency PPP ambiguity resolution with multi-constellation GNSS: BDS and Galileo. J. Geod. 2019, 93, 1105-1122. [CrossRef]

8. Geng, J.; Guo, J.; Meng, X.; Gao, K. Speeding up PPP ambiguity resolution using triple-frequency GPS/BeiDou/Galileo/QZSS data. J. Geod. 2020, 94. [CrossRef]

9. Ge, M.; Gendt, G.; Rothacher, M.; Shi, C.; Liu, J. Resolution of GPS carrier-phase ambiguities in Precise Point Positioning (PPP) with daily observations. J. Geod. 2008, 82, 389-399. [CrossRef]

10. Schaer, S. Differential code biases (DCB) in GNSS analysis. In Proceedings of the IGS Workshop, Miami Beach, FL, USA, 2-6 June 2008.

11. Montenbruck, O.; Hauschild, A.; Steigenberger, P. Differential Code Bias estimation using multi-GNSS observations and global ionosphere maps. Navigation 2014, 61, 191-201. [CrossRef]

12. Wang, N.; Yuan, Y.; Li, Z.; Montenbruck, O. Determination of Differential Code Biases with multi-GNSS observations. J. Geod. 2016, 90, 209-228. [CrossRef]

13. Montenbruck, O.; Hauschild, A. Code biases in multi-GNSS point positioning. In Proceedings of the ION ITM 2013, San Diego, CA, USA, 28-30 January 2013.

14. Schaer, S. SINEX_BIAS-Solution (Software/technique) Independent Exchange Format for GNSS Biases Version 1.00. In Proceedings of the IGS Workshop on GNSS Biases, Bern, Switzerland, 5-6 November 2015.

15. Villiger, A.; Schaer, S.; Dach, R.; Prange, L.; Sušnik, A.; Jäggi, A. Determination of GNSS pseudo-absolute code biases and their long-term combination. J. Geod. 2019, 93, 1487-1500. [CrossRef]

16. Montenbruck, O.; Hauschild, A.; Steigenberger, P.; Langley, R.B. Three's the challenge: A close look a GPS SVN62 triple-frequency signal combinations finds carrier-phase variations on the new L5. GPS World 2010, 21, 8-19.

17. Guo, J.; Geng, J. GPS satellite clock determination in case of inter-frequency clock biases for triple-frequency precise point positioning. J. Geod. 2018, 92, 1133-1142. [CrossRef]

18. Zhang, B.; Teunissen, P.J.G.; Odijk, D. A novel un-differenced PPP-RTK concept. J. Navig. 2011, 64, S180-S191. [CrossRef]

19. Odijk, D.; Zhang, B.; Khodabandeh, A.; Odolinski, R.; Teunissen, P.J.G. On the estimability of parameters in undifferenced, uncombined GNSS network and PPP-RTK user models by means of S-system theory. J. Geod. 2016, 90, 15-44. [CrossRef]

20. Zhang, B.; Chen, Y.; Yuan, Y. PPP-RTK based on undifferenced and uncombined observations: Theoretical and practical aspects. J. Geod. 2019, 93, 1011-1024. [CrossRef]

21. Liu, G.; Zhang, X.; Li, P. Estimating multi-frequency satellite phase biases of BeiDou using maximal decorrelated linear ambiguity combinations. GPS Solut. 2019, 23, 42. [CrossRef]

22. Montenbruck, O.; Hugentobler, U.; Dach, R.; Steigenberger, P.; Hauschild, A. Apparent clock variations of the Block IIF-1 (SVN62) GPS satellite. GPS Solut. 2011, 16, 303-313. [CrossRef]

23. Pan, L.; Zhang, X.; Guo, F.; Liu, J. GPS inter-frequency clock bias estimation for both uncombined and ionospheric-free combined triple-frequency precise point positioning. J. Geod. 2019, 93, 473-487. [CrossRef]

24. Zhao, L.; Ye, S.; Song, J. Handling the satellite inter-frequency biases in triple-frequency observations. Adv. Space. Res. 2017, 59, 2048-2057. [CrossRef]

25. Gong, X.; Gu, S.; Lou, Y.; Zheng, F.; Yang, X.; Wang, Z.; Liu, J. Research on empirical correction models of GPS Block IIF and BDS satellite inter-frequency clock bias. J. Geod. 2020, 94. [CrossRef]

26. Guo, F.; Zhang, X.; Wang, J. Timing group delay and differential code bias corrections for BeiDou positioning. J. Geod. 2015, 89, 427-445. [CrossRef]

27. Laurichesse, D. Carrier-phase ambiguity resolution: Handling the biases for improved triple-frequency PPP convergence. GPS World. 2015, 26, 49-54.

28. Li, H.; Li, B.; Xiao, G.; Wang, J.; Xu, T. Improved method for estimating the inter-frequency satellite clock bias of triple-frequency GPS. GPS Solut. 2016, 20, 751-760. [CrossRef] 
29. Teunissen, P.J.G. The least-squares ambiguity decorrelation adjustment: A method for fast GPS integer ambiguity estimation. J. Geod. 1995, 70, 65-82. [CrossRef]

30. De Jonge, P.; Teunissen, P.J.G.; Jonkman, N.; Joosten, P. The distributional dependence of the range on triple frequency GPS ambiguity resolution. In Proceedings of the National Technical Meeting of the Institute of Navigation, Anaheim, CA, USA, 26-28 January 2000; Volume 5, pp. 605-612.

31. Petit, G.; Luzum, B. IERS Conventions; IERS technical note No. 36; Publisher of the Federal Agency for Cartography and Geodesy: Frankfurt am Main, Germany, 2010; p. 179.

32. Wu, J.T.; Wu, S.C.; Hajj, G.A.; Bertiger, W.I.; Lichten, S.M. Effects of antenna orientation on GPS carrier phase. Manuscr. Geod. 1993, 18, 91-98.

33. Li, P.; Zhang, X.; Ren, X.; Zuo, X.; Pan, Y. Generating GPS satellite fractional cycle bias for ambiguity-fixed precise point positioning. GPS Solut. 2016, 20, 771-782. [CrossRef]

34. Hu, J.; Zhang, X.; Li, P.; Ma, F.; Pan, L. Multi-GNSS fractional cycle bias products generation for GNSS ambiguity-fixed PPP at Wuhan University. GPS Solut. 2020. [CrossRef]

(C) 2020 by the authors. Licensee MDPI, Basel, Switzerland. This article is an open access article distributed under the terms and conditions of the Creative Commons Attribution (CC BY) license (http://creativecommons.org/licenses/by/4.0/). 